\title{
EPSILON-STRONGLY GRADED RINGS, SEPARABILITY AND SEMISIMPLICITY
}

\author{
PATRIK NYSTEDT \\ Department of Engineering Science, University West, SE-46186 Trollhättan, Sweden \\ JOHAN ÖINERT \\ Department of Mathematics and Natural Sciences, Blekinge Institute of Technology, \\ SE-37179 Karlskrona, Sweden
}

\author{
HÉCTOR PINEDO \\ Escuela de Matemáticas, Universidad Industrial de Santander, Carrera 27 Calle 9, Edificio \\ Camilo Torres Apartado de correos 678, Bucaramanga, Colombia
}

\begin{abstract}
We introduce the class of epsilon-strongly graded rings and show that it properly contains both the class of strongly graded rings and the class of unital partial crossed products. We determine precisely when an epsilon-strongly graded ring is separable over its principal component. Thereby, we simultaneously generalize a result for strongly group graded rings by Năstăsescu, Van den Bergh and Van Oystaeyen, and a result for unital partial crossed products by Bagio, Lazzarin and Paques. We also show that the class of unital partial crossed products appear in the class of epsilon-strongly graded rings in a fashion similar to how the classical crossed products present themselves in the class of strongly graded rings. Thereby, we obtain, in the special case of unital partial crossed products, a short proof of a general result by Dokuchaev, Exel and Simón concerning when graded rings can be presented as partial crossed products. We also provide some interesting classes of examples of separable epsilon-strongly graded rings, with finite as well as infinite grading groups. In particular, we obtain an answer to a question raised by Le Bruyn, Van den Bergh and Van Oystaeyen in 1988.
\end{abstract}

\section{INTRODUCTION}

Let $S$ be an associative ring equipped with a non-zero multiplicative identity element 1 . Let $S / R$ be a ring extension. By this we mean that $R$ is a subring of $S$ containing 1 . Recall that $S / R$ is called separable if the multiplication map $m: S \otimes_{R} S \rightarrow S$ is a splitting epimorphism of $R$-bimodules. Equivalently, this can be formulated by saying that there is $x \in S \otimes_{R} S$

E-mail addresses: patrik.nystedt@hv.se; johan.oinert@bth.se; hpinedot@uis.edu.co. 2010 Mathematics Subject Classification. 16W50, 16S35, 16H05, 16K99, 16E60.

Key words and phrases. group graded ring, partial crossed product, separable, semisimple, Frobenius. 
satisfying $m(x)=1$ and that, for every $s \in S$, the relation $s x=x s$ holds. In that case, $x$ is called a separability element of $S \otimes_{R} S$. Separable ring extensions are a natural generalization of the classical separability condition for algebras over fields which in turn is a generalization of separable field extensions (see e.g. [7]). Năstăsescu, Van den Bergh and Van Oystaeyen [21] have generalized this even further by introducing the notion of a separable functor. They show that a ring extension is separable precisely when the associated restriction functor is separable. A lot of work has been devoted to the question of when ring extensions are separable (see e.g. [1], 14], 2], 3], 7], [9], 10], 15], [16], 19], 21], 23] and [24]). One reason for this intense interest is that some properties of the ground $\operatorname{ring} R$ automatically are inherited by $S$, such as semisimplicity and hereditarity (see e.g. [21]).

In the context of group graded rings, necessary and sufficient criteria for separability has been obtained in two different cases (see Theorem 1 and Theorem 2 below). Indeed, let $G$ be a group with identity element $e$. Let $S$ be graded by $G$. Recall that this means that, for all $g, h \in G$, there is an additive subgroup $S_{g}$ of $S$ such that $S=\oplus_{g \in G} S_{g}$ and $S_{g} S_{h} \subseteq S_{g h}$. The subring $R=S_{e}$ is called the principal component of $S$.

In the first case, $S$ is strongly graded. Recall that this means that $S_{g} S_{h}=S_{g h}$, for all $g, h \in G$. This makes each $S_{g}$, for $g \in G$, an invertible $R$-bimodule which implies that there is a unique ring automorphism $\beta_{g}: Z(R) \rightarrow Z(R)$ such that $\beta_{g}(r) s=s r$, for $r \in Z(R)$ and $s \in S_{g}$ (see [19] or e.g. Definition 9 and Proposition 12). If $G$ is finite, then the trace function $\operatorname{tr}_{\beta}: Z(R) \rightarrow Z(R)$ is defined by $\operatorname{tr}_{\beta}(r)=\sum_{g \in G} \beta_{g}(r)$, for $r \in Z(R)$.

Theorem 1 (Năstăsescu, Van den Bergh and Van Oystaeyen [21]). If $S$ is strongly graded by $G$, then $S / R$ is separable if and only if $G$ is finite and $1 \in \operatorname{tr}_{\beta}(Z(R))$.

In the second case, $S$ is a unital partial crossed product of $G$ over $R$. Recall that a unital twisted partial action of $G$ on $R$ is a triple

$$
\alpha=\left(\left\{D_{g}\right\}_{g \in G},\left\{\alpha_{g}\right\}_{g \in G},\left\{w_{g, h}\right\}_{(g, h) \in G \times G}\right)
$$

where for each $g \in G, D_{g}$ is a unital ideal of $R$ having an (not necessarily non-zero) identity element $1_{g}$ which is central in $R, \alpha_{g}: D_{g^{-1}} \rightarrow D_{g}$ is an isomorphism of rings, and for each $(g, h) \in G \times G, w_{g, h}$ is an invertible element from $D_{g} D_{g h}$, satisfying the following assertions for all $g, h, l \in G$ :

(P1) $\alpha_{e}=\mathrm{id}_{R}$;

(P2) $\alpha_{g}\left(D_{g^{-1}} D_{h}\right)=D_{g} D_{g h}$;

(P3) if $r \in D_{h^{-1}} D_{(g h)^{-1}}$, then $\alpha_{g}\left(\alpha_{h}(r)\right)=w_{g, h} \alpha_{g h}(r) w_{g, h}^{-1}$;

(P4) $w_{e, g}=w_{g, e}=1_{g}$;

(P5) if $r \in D_{g^{-1}} D_{h} D_{h l}$, then $\alpha_{g}\left(r w_{h, l}\right) w_{g, h l}=\alpha_{g}(r) w_{g, h} w_{g h, l}$.

Given a unital twisted partial action of $G$ on $R$, the unital partial crossed product $R \star_{\alpha}^{w} G$ is the direct sum $\oplus_{g \in G} D_{g} \delta_{g}$, in which the $\delta_{g}$ 's are formal symbols, and the multiplication is defined by the biadditive extension of the relations

(P6) $\left(r \delta_{g}\right)\left(r^{\prime} \delta_{h}\right)=r \alpha_{g}\left(r^{\prime} 1_{g^{-1}}\right) w_{g, h} \delta_{g h}$,

for $g, h \in G, r \in D_{g}$ and $r^{\prime} \in D_{h}$. If $G$ is finite, then the trace function $\operatorname{tr}_{\alpha}: Z(R) \rightarrow Z(R)$ is defined by $\operatorname{tr}_{\alpha}(r)=\sum_{g \in G} \alpha_{g}\left(r 1_{g^{-1}}\right)$, for $r \in Z(R)$.

Theorem 2 (Bagio, Lazzarin and Paques [1]). If $S$ is a unital partial crossed product of a finite group $G$ over $R$, then $S / R$ is separable if and only if $1 \in \operatorname{tr}_{\alpha}(Z(R))$. 
In this article, we wish to unify Theorem 1 and Theorem 2 (see Theorem 3) for a class of rings which properly contains both the class of strongly graded rings and the class of partial crossed products. We call this class of rings epsilon-strongly graded. The term "epsilonstrongly" is supposed to be suggestive of the fact that the grading is "an epsilon away" from being strong. Let us briefly describe the idea behind this class of rings. Suppose that $S$ is a ring graded by a group $G$ and take $g, h \in G$. Instead of postulating that $S_{g} S_{h}=S_{g h}$, as in the strongly graded case, we relax this condition by saying that $S_{g} S_{g^{-1}}$ and $S_{h^{-1}} S_{h}$ are unital ideals of $R$ such that the equalities $S_{g} S_{h}=S_{g} S_{g^{-1}} S_{g h}=S_{g h} S_{h^{-1}} S_{h}$ hold. The multiplicative identity element in $S_{g} S_{g^{-1}}$ is denoted by $\epsilon_{g}$. Here is an outline of the article.

In Section 2, we introduce epsilon-strongly graded rings (see Definition 4) and we give several equivalent characterizations of them (see Proposition 17).

In Section 3, we show that if $S$ is epsilon-strongly graded by $G$, then we can define a trace function $\operatorname{tr}_{\gamma}: Z(R)_{\text {fin }} \rightarrow Z(R)$ (see Definition 14) which generalizes the trace functions from both the strongly graded case and the partial crossed product situation. Here $Z(R)_{\text {fin }}$ is the set of $r \in Z(R)$ with the property that for all but finitely many $g \in G$, the relation $r \epsilon_{g}=0$ holds. At the end of Section 3, we show the following simultaneous generalization of Theorem 1 and Theorem 2, Notice that our result holds for any, possibly infinite, group $G$.

Theorem 3. If $S$ is epsilon-strongly graded by $G$, then $S / R$ is separable if and only if $1 \in$ $\operatorname{tr}_{\gamma}\left(Z(R)_{\text {fin }}\right)$.

In Section 4, we use Theorem 3 to find criteria for when epsilon-strongly graded rings are semisimple, hereditary or Frobenius (see Theorem 23 and Theorem 24).

In Section 5, we show that a result concerning simplicity for strongly graded rings from [17. Theorem 6.6] can be generalized to epsilon-strongly graded rings (see Proposition 291).

In Section 6, we introduce epsilon-crossed products (see Definition 32). We show that the class of epsilon-crossed products coincides with the class of unital partial crossed products (see Theorem 33). This is an epsilon-analogue of how the classical crossed products appear in the class of strongly graded rings (see e.g. [20]). Thereby, we obtain, in the special case of unital partial crossed products, a short proof of a general result by Dokuchaev, Exel and Simón [8] concerning when graded rings can be presented as partial crossed products. At the end of the section, we use Theorem 3 to reformulate Theorem 2 so that it holds for any, possibly infinite, group $G$ (see Theorem 35).

In Section 7, we provide a class of examples of separable epsilon-strongly $\mathbb{Z}_{2}$-graded rings, neither of which are strongly graded, nor partial crossed products, in any natural way (see Proposition 38 and Proposition [39). Thereby, we provide the first known non-trivial example of a ring, graded by a finite group, which is separable over its principal component but yet not strongly graded (see Remark 40 and [14, Remark II.5.1.6]).

In Section 8, we consider Morita rings which are in a natural way $\mathbb{Z}$-graded. We show that, under weak assumptions, they are in fact epsilon-strongly graded and separable over their principal components (see Proposition 42).

\section{Some Characterizations of Epsilon-Strongly Graded Rings}

In this section, we introduce epsilon-strongly graded rings (see Definition 44) and we give several equivalent characterizations of them (see Proposition 7 ). Throughout the rest of this article, unless otherwise stated, let $G$ be an arbitrary group with identity element $e$. In this section, let $S$ be an arbitrary unital ring which is graded by $G$ and put $R=S_{e}$. 
Definition 4. Let $S$ be a ring which is graded by $G$. We say that $S$ is epsilon-strongly graded by $G$ if for each $g \in G, S_{g} S_{g^{-1}}$ is a unital ideal of $R$ such that for all $g, h \in G$ the equalities $S_{g} S_{h}=S_{g} S_{g^{-1}} S_{g h}=S_{g h} S_{h^{-1}} S_{h}$ hold. In that case, for each $g \in G$, we let $\epsilon_{g}$ denote the multiplicative identity element of $S_{g} S_{g^{-1}}$.

Proposition 5. If $S$ is epsilon-strongly graded by $G$, then, for every $g \in G, \epsilon_{g} \in Z(R)$.

Proof. Take $g \in G$ and $r \in R$. Since $S_{g} S_{g^{-1}}$ is an $R$-ideal it follows that $\epsilon_{g} r, r \epsilon_{g} \in S_{g} S_{g^{-1}}$. Using that $\epsilon_{g}$ is a multiplicative identity element of $S_{g} S_{g^{-1}}$, we therefore get that $\epsilon_{g} r=$ $\left(\epsilon_{g} r\right) \epsilon_{g}=\epsilon_{g}\left(r \epsilon_{g}\right)=r \epsilon_{g}$.

Definition 6. Following [4, Definition 4.5] we say that $S$ is symmetrically graded if for every $g \in G$, the equality $S_{g} S_{g^{-1}} S_{g}=S_{g}$ holds.

Proposition 7. The following assertions are equivalent:

(i) $S$ is epsilon-strongly graded by $G$;

(ii) $S$ is symmetrically graded by $G$, and for every $g \in G$ the $R$-ideal $S_{g} S_{g^{-1}}$ is unital;

(iii) For every $g \in G$ there is an element $\epsilon_{g} \in S_{g} S_{g^{-1}}$ such that for all $s \in S_{g}$ the relations $\epsilon_{g} s=s=s \epsilon_{g^{-1}}$ hold;

(iv) For every $g \in G$ the left $R$-module $S_{g}$ is finitely generated and projective, and the map $n_{g}:\left(S_{g}\right)_{R} \rightarrow \operatorname{Hom}_{R}\left({ }_{R} S_{g^{-1}}, R\right)_{R}$, defined by $n_{g}(s)(t)=t s$, for $s \in S_{g}$ and $t \in S_{g^{-1}}$, is an isomorphism of right $R$-modules.

Proof. (i) $\Rightarrow$ (ii): This follows immediately from Definition 4 by putting $h=e$.

(ii) $\Rightarrow$ (iii): Take $g \in G$ and $s \in S_{g}$. Let $\epsilon_{g}$ denote the multiplicative identity element of $S_{g} S_{g^{-1}}$. Using that $S$ is symmetrically graded, we may write $s=\sum_{i=1}^{n} a_{i} b_{i} c_{i}$ where $a_{1}, \ldots, a_{n}, c_{1}, \ldots, c_{n} \in S_{g}$ and $b_{1}, \ldots, b_{n} \in S_{g^{-1}}$. This yields

$$
\epsilon_{g} s=\sum_{i=1}^{n} \epsilon_{g} \underbrace{a_{i} b_{i}}_{\in S_{g} S_{g}-1} c_{i}=\sum_{i=1}^{n} a_{i} b_{i} c_{i}=s
$$

and similarly

$$
s \epsilon_{g^{-1}}=\sum_{i=1}^{n} a_{i} \underbrace{b_{i} c_{i}}_{\in S_{g^{-1}} S_{g}} \epsilon_{g^{-1}}=\sum_{i=1}^{n} a_{i} b_{i} c_{i}=s .
$$

(iii) $\Rightarrow($ i): Take $g, h \in G$. Then it follows that

$$
S_{g} S_{h}=\epsilon_{g} S_{g} S_{h} \subseteq S_{g} S_{g^{-1}} S_{g} S_{h} \subseteq S_{g} S_{g^{-1}} S_{g h} \subseteq S_{g} S_{h}
$$

and

$$
S_{g} S_{h}=S_{g} S_{h} \epsilon_{h^{-1}} \subseteq S_{g} S_{h} S_{h^{-1}} S_{h} \subseteq S_{g h} S_{h^{-1}} S_{h}=S_{g} S_{h}
$$

It is clear that $\epsilon_{g}$ is a multiplicative identity element of $S_{g} S_{g^{-1}}$.

(iii) $\Rightarrow$ (iv): Suppose that (iii) holds. From the relation $\epsilon_{g^{-1}} \in S_{g^{-1}} S_{g}$ it follows that there is $n \in \mathbb{N}$ and $u_{i} \in S_{g^{-1}}$ and $v_{i} \in S_{g}$, for $i \in\{1, \ldots, n\}$, such that $\sum_{i=1}^{n} u_{i} v_{i}=\epsilon_{g^{-1}}$. For each $i \in\{1, \ldots, n\}$, define the left $R$-linear map $f_{i}: S_{g} \rightarrow R$ by the relations $f_{i}(s)=s u_{i}$, for $s \in S_{g}$. Take $s \in S_{g}$. Then $s=s \epsilon_{g^{-1}}=\sum_{i=1}^{n} s u_{i} v_{i}=\sum_{i=1}^{n} f_{i}(s) v_{i}$. Therefore $\left\{v_{i}\right\}_{i=1}^{n}$ and $\left\{f_{i}\right\}_{i=1}^{n}$ form "dual bases" and $S_{g}$ is therefore finitely generated and projective as a left $R$ module. Next we show that $n_{g}$ is a monomorphism. Suppose that $s \in S_{g}$ satisfies $n_{g}(s)=0$. Then $s=\epsilon_{g} s \in S_{g} S_{g^{-1}} s=S_{g} n_{g}(s)\left(S_{g^{-1}}\right)=\{0\}$. Therefore $s=0$. Now we show that $n_{g}$ is surjective. There is $n \in \mathbb{N}$ and $a_{i} \in S_{g}$ and $v_{i} \in S_{g^{-1}}$, for $i \in\{1, \ldots, n\}$, such that $\sum_{i=1}^{n} a_{i} b_{i}=$ 
$\epsilon_{g}$. For each $i \in\{1, \ldots, n\}$, define the left $R$-linear map $g_{i}: S_{g^{-1}} \rightarrow R$ by the relations $g_{i}(s)=s a_{i}$, for $s \in S_{g^{-1}}$. Take $t \in S_{g^{-1}}$. Then $t=t \epsilon_{g}=\sum_{i=1}^{n} t a_{i} b_{i}=\sum_{i=1}^{n} g_{i}(t) b_{i}$. Take $f \in \operatorname{Hom}_{R}\left({ }_{R} S_{g^{-1}}, R\right)_{R}$. Then $f(t)=\sum_{i=1}^{n} g_{i}(t) f\left(b_{i}\right)=t \sum_{i=1}^{n} a_{i} f\left(b_{i}\right)=n_{g}\left(\sum_{i=1}^{n} a_{i} f\left(b_{i}\right)\right)(t)$. Therefore, $f=n_{g}\left(\sum_{i=1}^{n} a_{i} f\left(b_{i}\right)\right)$. Hence, $n_{g}$ is surjective.

(iv) $\Rightarrow$ (iii): Take $a \in S_{g}$ and $b \in S_{g^{-1}}$. Suppose that the left $R$-module $S_{g}$ is finitely generated and projective, and that the map $n_{g}$ is an isomorphism of right $R$-modules. The dual basis lemma shows that there are $b_{1}, \ldots, b_{n} \in S_{g^{-1}}$ and $f_{1}, \ldots, f_{n} \in \operatorname{Hom}_{R}\left(S_{g^{-1}}, R\right)$ such that $b=\sum_{i=1}^{n} f_{i}(b) b_{i}$. For every $i \in\{1, \ldots, n\}$, there is $a_{i} \in S_{g}$ such that $n_{g}\left(a_{i}\right)=f_{i}$. Hence $b=\sum_{i=1}^{n} n_{g}\left(a_{i}\right)(b) b_{i}=\sum_{i=1}^{n} b a_{i} b_{i}=b \epsilon_{g}$, where $\epsilon_{g}=\sum_{i=1}^{n} a_{i} b_{i} \in S_{g} S_{g^{-1}}$. This shows that $S_{g^{-1}}\left(1-\epsilon_{g}\right)=\{0\}$. Therefore $S_{g^{-1}}\left(1-\epsilon_{g}\right) a=\{0\}$ and thus $n_{g}\left(\left(1-\epsilon_{g}\right) a\right)\left(S_{g^{-1}}\right)=\{0\}$. This implies that $n_{g}\left(\left(1-\epsilon_{g}\right) a\right)=0$. But since $n_{g}$ is injective, we finally get that $\left(1-\epsilon_{g}\right) a=0$ and hence $a=\epsilon_{g} a$. Therefore $S$ is epsilon-strongly graded by $G$.

Proposition 8. If $S$ is epsilon-strongly graded by $G$, then $S$ is strongly graded by $G$ if and only if for every $g \in G$ the equality $\epsilon_{g}=1$ holds.

Proof. Suppose that $S$ is strongly graded by $G$. Take $g \in G$. Since $S_{g} S_{g^{-1}}=R$, we get that $\epsilon_{g}=1$. Now suppose that $S$ is epsilon-strongly graded with $\epsilon_{g}=1$, for all $g \in G$. Since $S_{g} S_{g^{-1}}$ is a unital ideal of $R$ with 1 as a multiplicative identity, it follows that $R=R 1 \subseteq R S_{g} S_{g^{-1}} \subseteq R$. Therefore $S_{g} S_{g^{-1}}=R$.

\section{Separability}

In this section, we shall assume that $S$ is an arbitrary unital ring which is epsilon-strongly graded by $G$. We will, for each $g \in G$, introduce an additive function $\gamma_{g}: S \rightarrow S$ (see Definition (9). These functions will in turn be used to define a trace function $\operatorname{tr}_{\gamma}: Z(R)_{\text {fin }} \rightarrow$ $Z(R)$ (see Definition 14). At the end of this section, we prove Theorem 3. Let $\mathbb{N}$ denote the set of positive integers.

Definition 9. Let $g \in G$ be arbitrary. From the relation $\epsilon_{g} \in S_{g} S_{g^{-1}}$ it follows that there is $n_{g} \in \mathbb{N}$, and $u_{g}^{(i)} \in S_{g}$ and $v_{g^{-1}}^{(i)} \in S_{g^{-1}}$, for $i \in\left\{1, \ldots, n_{g}\right\}$, such that $\sum_{i=1}^{n_{g}} u_{g}^{(i)} v_{g^{-1}}^{(i)}=\epsilon_{g}$. Unless otherwise stated, the elements $u_{g}^{(i)}$ and $v_{g^{-1}}^{(i)}$ are fixed. We also assume that $n_{e}=1$ and $u_{e}^{(1)}=v_{e}^{(1)}=1$. Define the additive function $\gamma_{g}: S \rightarrow S$ by $\gamma_{g}(s)=\sum_{i=1}^{n_{g}} u_{g}^{(i)} s v_{g}^{(i)}$, for $s \in S$.

Proposition 10. For any $g \in G$ and $r \in Z(R)$, the definition of $\gamma_{g}(r)$ does not depend on the choice of the elements $u_{g}^{(i)}$ and $v_{g^{-1}}^{(i)}$.

Proof. Take $m_{g} \in \mathbb{N}, s_{g}^{(j)} \in S_{g}$ and $t_{g^{-1}}^{(j)} \in S_{g^{-1}}$, for $j \in\left\{1, \ldots, m_{g}\right\}$, such that $\sum_{j=1}^{m_{g}} s_{g}^{(j)} t_{g^{-1}}^{(j)}=$ $\epsilon_{g}$. Then

$$
\gamma_{g}(r)=\sum_{i=1}^{n_{g}} u_{g}^{(i)} r v_{g^{-1}}^{(i)}=\sum_{i=1}^{n_{g}} \epsilon_{g} u_{g}^{(i)} r v_{g^{-1}}^{(i)}=\sum_{i=1}^{n_{g}} \sum_{j=1}^{m_{g}} s_{g}^{(j)} t_{g^{-1}}^{(j)} u_{g}^{(i)} r v_{g^{-1}}^{(i)} .
$$

Since $t_{g^{-1}}^{(j)} u_{g}^{(i)} \in R$ and $r \in Z(R)$, the last sum equals

$$
\sum_{i=1}^{n_{g}} \sum_{j=1}^{m_{g}} s_{g}^{(j)} r t_{g^{-1}}^{(j)} u_{g}^{(i)} v_{g^{-1}}^{(i)}=\sum_{j=1}^{m_{g}} s_{g}^{(j)} r t_{g^{-1}}^{(j)} \epsilon_{g}=\sum_{j=1}^{m_{g}} s_{g}^{(j)} r t_{g^{-1}}^{(j)} .
$$


Proposition 11. For any $g, h \in G$ and $r \in Z(R), \gamma_{g}\left(\gamma_{h}(r)\right)=\gamma_{g h}(r) \epsilon_{g}$ holds.

Proof. From the definitions of $\gamma_{g}$ and $\gamma_{h}$, it follows that

$$
\gamma_{g}\left(\gamma_{h}(r)\right)=\sum_{i=1}^{n_{h}} \gamma_{g}\left(u_{h}^{(i)} r v_{h^{-1}}^{(i)}\right)=\sum_{i=1}^{n_{h}} \sum_{j=1}^{n_{g}} u_{g}^{(j)} u_{h}^{(i)} r v_{h^{-1}}^{(i)} v_{g^{-1}}^{(j)}
$$

Since $u_{g}^{(j)} u_{h}^{(i)} \in S_{g h}$, the last sum equals

$$
\sum_{i=1}^{n_{h}} \sum_{j=1}^{n_{g}} \epsilon_{g h} u_{g}^{(j)} u_{h}^{(i)} r v_{h^{-1}}^{(i)} v_{g^{-1}}^{(j)}=\sum_{i=1}^{n_{h}} \sum_{j=1}^{n_{g}} \sum_{k=1}^{n_{g h}} u_{g h}^{(k)} v_{h^{-1} g^{-1}}^{(k)} u_{g}^{(j)} u_{h}^{(i)} r v_{h^{-1}}^{(i)} v_{g^{-1}}^{(j)} .
$$

Using that $r \in Z(R)$ and $v_{h^{-1} g^{-1}}^{(k)} u_{g}^{(j)} u_{h}^{(i)} \in R$, the last sum equals

$$
\sum_{i=1}^{n_{h}} \sum_{j=1}^{n_{g}} \sum_{k=1}^{n_{g h}} u_{g h}^{(k)} r v_{h^{-1} g^{-1}}^{(k)} u_{g}^{(j)} u_{h}^{(i)} v_{h^{-1}}^{(i)} v_{g^{-1}}^{(j)}=\sum_{j=1}^{n_{g}} \sum_{k=1}^{n_{g h}} u_{g h}^{(k)} r v_{h^{-1} g^{-1}}^{(k)} u_{g}^{(j)} \epsilon_{h} v_{g^{-1}}^{(j)}
$$

Since $v_{h^{-1} g^{-1}}^{(k)} u_{g}^{(j)} \in S_{h^{-1}}$, the last sum equals

$$
\sum_{j=1}^{n_{g}} \sum_{k=1}^{n_{g h}} u_{g h}^{(k)} r v_{h^{-1} g^{-1}}^{(k)} u_{g}^{(j)} v_{g^{-1}}^{(j)}=\sum_{k=1}^{n_{g h}} u_{g h}^{(k)} r v_{h^{-1} g^{-1}}^{(k)} \epsilon_{g}=\gamma_{g h}(r) \epsilon_{g} .
$$

Proposition 12. Let $g \in G$ be arbitrary. The additive function $\gamma_{g}: S \rightarrow S$ restricts to a surjective ring homomorphism $Z(R) \rightarrow Z(R) \epsilon_{g}$ which satisfies the relation $\gamma_{g}(r) s_{g}=s_{g} r$, for all $r \in Z(R), s_{g} \in S_{g}$. This function, in turn, restricts to a ring isomorphism $Z(R) \epsilon_{g^{-1}} \rightarrow$ $Z(R) \epsilon_{g}$.

Proof. First we show that $\gamma_{g}(Z(R)) \subseteq Z(R) \epsilon_{g}$. From the definition of $\gamma_{g}$ it follows that $\gamma_{g}(S) \subseteq S \epsilon_{g}$. Thus, since $\epsilon_{g}$ is idempotent, we only need to show that $\gamma_{g}(Z(R)) \subseteq Z(R)$. To this end, take $r \in Z(R)$ and $r^{\prime} \in R$. Then, since $v_{g^{-1}}^{(i)} r^{\prime} \in S_{g^{-1}}$, we get that

$$
\gamma_{g}(r) r^{\prime}=\sum_{i=1}^{n_{g}} u_{g}^{(i)} r v_{g^{-1}}^{(i)} r^{\prime}=\sum_{i=1}^{n_{g}} u_{g}^{(i)} r v_{g^{-1}}^{(i)} r^{\prime} \epsilon_{g}=\sum_{i=1}^{n_{g}} \sum_{j=1}^{n_{g}} u_{g}^{(i)} r v_{g^{-1}}^{(i)} r^{\prime} u_{g}^{(j)} v_{g^{-1}}^{(j)}
$$

Using that $r \in Z(R), v_{g^{-1}}^{(i)} r^{\prime} u_{g}^{(j)} \in R$ and $r^{\prime} u_{g}^{(j)} \in S_{g}$, the last sum equals

$$
\sum_{i=1}^{n_{g}} \sum_{j=1}^{n_{g}} u_{g}^{(i)} v_{g^{-1}}^{(i)} r^{\prime} u_{g}^{(j)} r v_{g^{-1}}^{(j)}=\sum_{j=1}^{n_{g}} \epsilon_{g} r^{\prime} u_{g}^{(j)} r v_{g^{-1}}^{(j)}=\sum_{j=1}^{n_{g}} r^{\prime} u_{g}^{(j)} r v_{g^{-1}}^{(j)}=r^{\prime} \gamma_{g}(r) .
$$

This shows that $\gamma_{g}(r) \in Z(R)$. Now we show that the restriction of $\gamma_{g}$ to $Z(R)$ respects multiplication. Take $r, r^{\prime} \in Z(R)$. Then

$$
\gamma_{g}\left(r r^{\prime}\right)=\sum_{i=1}^{n_{g}} u_{g}^{(i)} r r^{\prime} v_{g^{-1}}^{(i)}=\sum_{i=1}^{n_{g}} \epsilon_{g} u_{g}^{(i)} r r^{\prime} v_{g^{-1}}^{(i)}=\sum_{i=1}^{n_{g}} \sum_{j=1}^{n_{g}} u_{g}^{(j)} v_{g^{-1}}^{(j)} u_{g}^{(i)} r r^{\prime} v_{g^{-1}}^{(i)}
$$


Since $r \in Z(R)$ and $v_{g^{-1}}^{(j)} u_{g}^{(i)} \in R$, the last sum equals

$$
\sum_{i=1}^{n_{g}} \sum_{j=1}^{n_{g}} u_{g}^{(j)} r v_{g^{-1}}^{(j)} u_{g}^{(i)} r^{\prime} v_{g^{-1}}^{(i)}=\sum_{j=1}^{n_{g}} u_{g}^{(j)} r v_{g^{-1}}^{(j)} \sum_{i=1}^{n_{g}} u_{g}^{(i)} r^{\prime} v_{g^{-1}}^{(i)}=\gamma_{g}(r) \gamma_{g}\left(r^{\prime}\right) .
$$

Next, we show that the restriction $Z(R) \rightarrow Z(R) \epsilon_{g}$ is surjective. Take $r \in Z(R)$. From Proposition [1], we get that $\gamma_{g}\left(\gamma_{g^{-1}}\left(r \epsilon_{g}\right)\right)=\gamma_{e}\left(r \epsilon_{g}\right) \epsilon_{g}=r \epsilon_{g}^{2}=r \epsilon_{g}$. For any $s_{g} \in S_{g}$, using that $v_{g^{-1}}^{(i)} s_{g} \in R$, we conclude that

$$
\gamma_{g}(r) s_{g}=\sum_{i=1}^{n_{g}} u_{g}^{(i)} r v_{g^{-1}}^{(i)} s_{g}=\sum_{i=1}^{n_{g}} u_{g}^{(i)} v_{g^{-1}}^{(i)} s_{g} r=\epsilon_{g} s_{g} r=s_{g} r .
$$

Finally, we need to show that the restriction $Z(R) \epsilon_{g^{-1}} \rightarrow Z(R) \epsilon_{g}$ is injective. Suppose that $r \in Z(R)$ is chosen so that $\gamma_{g}\left(r \epsilon_{g^{-1}}\right)=0$. From Proposition [11, we get that $r \epsilon_{g^{-1}}=r \epsilon_{g^{-1}}^{2}=$ $\gamma_{e}\left(r \epsilon_{g^{-1}}\right) \epsilon_{g^{-1}}=\gamma_{g^{-1}}\left(\gamma_{g}\left(r \epsilon_{g^{-1}}\right)\right)=0$.

Remark 13. Notice that, by Proposition 11 and Proposition 12, the collection of (restriction) maps $\gamma_{g}: Z(R) \epsilon_{g^{-1}} \rightarrow Z(R) \epsilon_{g}$, for $g \in G$, yields a partial action of $G$ on $Z(R)$.

Definition 14. Let $Z(R)_{\text {fin }}$ denote the set of $r \in Z(R)$ such that for all but finitely many $g \in G$, the relation $r \epsilon_{g}=0$ holds. Define the trace function $\operatorname{tr}_{\gamma}: Z(R)_{\text {fin }} \rightarrow Z(R)$ by $\operatorname{tr}_{\gamma}(r)=\sum_{g \in G} \gamma_{g}(r)$, for $r \in R$.

Remark 15. Recall that if we for every $g \in G$ put

$$
\left(S \otimes_{R} S\right)_{g}=\bigoplus_{\substack{\left(g^{\prime}, g^{\prime \prime}\right) \in G \times G \\ g^{\prime} g^{\prime \prime}=g}} S_{g^{\prime}} \otimes_{R} S_{g^{\prime \prime}}
$$

then this defines a graded $R$-bimodule structure on $S \otimes_{R} S$. We will refer to this as the $G$-grading of $S \otimes_{R} S$. There is another type of grading on $S \otimes_{R} S$ that we will also use. If we, for every $(g, h) \in G \times G$, put

$$
\left(S \otimes_{R} S\right)_{(g, h)}=S_{g} \otimes_{R} S_{h},
$$

then this defines a graded additive structure on $S \otimes_{R} S$. We will refer to this as the $(G \times G)$ grading on $S \otimes_{R} S$. For more details concerning these gradings, see [20].

Proof of Theorem [3. First we show the "if" statement. Suppose that there is $c \in Z(R)_{\text {fin }}$ such that $\operatorname{tr}_{\gamma}(c)=1$. We wish to show that $S / R$ is separable. To this end, put $x=$ $\sum_{g \in G} \sum_{i=1}^{n_{g}} u_{g}^{(i)} c \otimes v_{g^{-1}}^{(i)}$. From the definition of $Z(R)_{\text {fin }}$ it follows that $x$ is well-defined, since for all but finitely many $g \in G$, we get that $u_{g}^{(i)} c=u_{g}^{(i)} \epsilon_{g^{-1}} c=u_{g}^{(i)} 0=0$. Now

$$
m(x)=\sum_{g \in G} \sum_{i=1}^{n_{g}} u_{g}^{(i)} c v_{g^{-1}}^{(i)}=\sum_{g \in G} \gamma_{g}(c)=\operatorname{tr}_{\gamma}(c)=1 .
$$


Next we show that $x$ commutes with all elements of $S$. To this end, take $h \in G$ and $s \in S_{h}$. Using that $s u_{g}^{(i)} \in S_{h g}$, we get that

$$
\begin{aligned}
s x & =\sum_{g \in G} \sum_{i=1}^{n_{g}} s u_{g}^{(i)} c \otimes v_{g^{-1}}^{(i)}=\sum_{g \in G} \sum_{i=1}^{n_{g}} \epsilon_{h g} s u_{g}^{(i)} c \otimes v_{g^{-1}}^{(i)} \\
& =\sum_{g \in G} \sum_{i=1}^{n_{g}} \sum_{j=1}^{n_{h g}} u_{h g}^{(j)} v_{g^{-1} h^{-1}}^{(j)} s u_{g}^{(i)} c \otimes v_{g^{-1}}^{(i)} .
\end{aligned}
$$

Since $c \in Z(R)$ and $v_{g^{-1} h^{-1}}^{(j)} s u_{g}^{(i)} \in R$, the last sum equals

$$
\sum_{g \in G} \sum_{i=1}^{n_{g}} \sum_{j=1}^{n_{h g}} u_{h g}^{(j)} c \otimes v_{g^{-1} h^{-1}}^{(j)} s u_{g}^{(i)} v_{g^{-1}}^{(i)}=\sum_{g \in G} \sum_{j=1}^{n_{h g}} u_{h g}^{(j)} c \otimes v_{g^{-1} h^{-1}}^{(j)} s \epsilon_{g} .
$$

Using that $v_{g^{-1} h^{-1}}^{(j)} s \in S_{g^{-1}}$, the last sum equals $\sum_{g \in G} \sum_{j=1}^{n_{h g}} u_{h g}^{(j)} c \otimes v_{g^{-1} h^{-1}}^{(j)} s=x s$. Therefore, $x$ is a separability element for $S / R$.

Now we show the "only if" statement. Suppose that $x \in S \otimes_{R} S$ is a separability element for $S / R$. Then $x$ satisfies $m(x)=1$ and, for each $s \in S$, the relation $x s=s x$ holds. From the $G$-grading on $S \otimes_{R} S$ it follows that there are unique $y \in\left(S \otimes_{R} S\right)_{e}$ and $z \in \oplus_{g \in G \backslash\{e\}}\left(S \otimes_{R} S\right)_{g}$ such that $x=y+z$. Then we get that $1=m(x)=m(y)+m(z)$. But since $1 \in R$ and $m(z) \in$ $\oplus_{g \in G \backslash\{e\}} S_{g}$, we get that $m(z)=0$ and $m(y)=1$. We claim that for each $s \in S$, the relation $y s=s y$ holds. To this end, take $h \in G$ and $s \in S_{h}$. Then $0=s x-x s=s y-y s+s z-z s$. Since $s y-y s \in\left(S \otimes_{R} S\right)_{h}$ and $s z-z s \in \sum_{g \in G \backslash\{e\}}\left(\left(S \otimes_{R} S\right)_{h g}+\left(S \otimes_{R} S\right)_{g h}\right)$, we get that $s y-y s=0$ and $s z-z s=0$. In particular, $y$ is also a separability element for $S / R$. Now, for each $g \in G$, there is $d_{g} \in S_{g} \otimes_{R} S_{g^{-1}}$ such that for all but finitely many $g \in G, d_{g}=0$, and $y=\sum_{g \in G} d_{g}$. Furthermore, for each $g \in G$, there is $l_{g} \in \mathbb{N}$, and $a_{g}^{(i)} \in S_{g}$ and $b_{g^{-1}}^{(i)} \in S_{g^{-1}}$, for $i \in\left\{1, \ldots, l_{g}\right\}$, such that $d_{g}=\sum_{i=1}^{l_{g}} a_{g}^{(i)} \otimes b_{g^{-1}}^{(i)}$. For each $g \in G$, put $c_{g}=m\left(d_{g}\right)$. Take $h \in G$. From the $(G \times G)$-grading on $S \otimes_{R} S$ it follows that $d_{h}$ commutes with every $r \in R$. Therefore, it follows that $c_{h} \in Z(R)$. Take $s \in S_{h}$. From the $(G \times G)$-grading on $S \otimes_{R} S$ it also follows that $s d_{e}=d_{h} s$. Applying $m$ gives us that $s c_{e}=c_{h} s$. In particular, we get that

$$
\gamma_{h}\left(c_{e}\right)=\sum_{i=1}^{n_{h}} u_{h}^{(i)} c_{e} v_{h^{-1}}^{(i)}=\sum_{i=1}^{n_{h}} c_{h} u_{h}^{(i)} v_{h^{-1}}^{(i)}=c_{h} \epsilon_{h}=\epsilon_{h} c_{h}=\epsilon_{h} m\left(d_{h}\right)=m\left(d_{h}\right)=c_{h} .
$$

Using that $d_{h}=0$, for all but finitely many $h \in G$, the same holds for $c_{h}$ and hence also for $\gamma_{h}\left(c_{e}\right)$. By summing over $h \in G$, we get that

$$
\operatorname{tr}_{\gamma}\left(c_{e}\right)=\sum_{h \in G} \gamma_{h}\left(c_{e}\right)=\sum_{h \in G} c_{h}=\sum_{h \in G} m\left(d_{h}\right)=m(y)=1 .
$$

Moreover, by Proposition 11 it follows that for all but finitely many $h \in G$, the relation $c_{e} \epsilon_{h^{-1}}=\gamma_{h^{-1}}\left(\gamma_{h}\left(c_{e}\right)\right)=0$ holds. Thus, $c_{e} \in Z(R)_{\mathrm{fin}}$.

Definition 16. Let $Z(R)_{\text {fin }}^{\gamma}$ denote the set of $r \in Z(R)_{\text {fin }}$ with the property that for every $g \in G$, the relation $\gamma_{g}(r)=r \epsilon_{g}$ holds.

Lemma 17. With the above notation, the following assertions hold:

(a) The set $Z(R)_{\text {fin }}$ is a (possibly non-unital) subring of $Z(R) .1 \in Z(R)_{\text {fin }}$ if and only if for all but finitely many $g \in G, \epsilon_{g}=0$. 
(b) The set $Z(R)_{\mathrm{fin}}^{\gamma}$ is a (possibly non-unital) subring of $Z(R)_{\mathrm{fin}} \cdot 1 \in Z(R)_{\mathrm{fin}}^{\gamma}$ if and only if for all but finitely many $g \in G, \epsilon_{g}=0$.

(c) $\operatorname{tr}_{\gamma}\left(Z(R)_{\text {fin }}\right) \subseteq Z(R)_{\text {fin }}^{\gamma}$ and the function $\operatorname{tr}_{\gamma}: Z(R)_{\text {fin }} \rightarrow Z(R)_{\text {fin }}^{\gamma}$ is a $Z(R)_{\text {fin }}^{\gamma}$-bimodule homomorphism.

(d) Suppose that $\epsilon_{g}=0$, for all but finitely many $g \in G$. If $r \in Z(R)_{\text {fin }}^{\gamma}$ and $r$ is invertible in $Z(R)$, then $r^{-1} \in Z(R)_{\text {fin }}^{\gamma}$.

Proof. (a): Take $r, r^{\prime} \in Z(R)_{\text {fin. }}$. Let $X(r)$ denote the finite set $\left\{g \in G \mid r \epsilon_{g} \neq 0\right\}$. Then $X\left(r r^{\prime}\right) \subseteq X\left(r^{\prime}\right)$ which is a finite set. Also $X\left(r+r^{\prime}\right) \subseteq X(r) \cup X\left(r^{\prime}\right)$ which is a finite set. Therefore $Z(R)_{\text {fin }}$ is a subring of $Z(R)$. Also $1 \in Z(R)_{\text {fin }}$ precisely when $\epsilon_{g}=1 \epsilon_{g}=0$ for all but finitely many $g \in G$.

(b): Take $r, r^{\prime} \in Z(R)_{\text {fin }}^{\gamma}$. From (a) we know that $r r^{\prime}, r+r^{\prime} \in Z(R)_{\text {fin. }}$. Take $g \in G$. From Proposition 12, it follows that $\gamma_{g}\left(r r^{\prime}\right)=\gamma_{g}(r) \gamma_{g}\left(r^{\prime}\right)=r \epsilon_{g} r^{\prime} \epsilon_{g}=r r^{\prime} \epsilon_{g}$. Also $\gamma_{g}\left(r+r^{\prime}\right)=$ $\gamma_{g}(r)+\gamma_{g}\left(r^{\prime}\right)=r \epsilon_{g}+r^{\prime} \epsilon_{g}=\left(r+r^{\prime}\right) \epsilon_{g}$. Therefore $r r^{\prime}, r+r^{\prime} \in Z(R)_{\text {fin }}^{\gamma}$. Since $\gamma_{g}(1)=\epsilon_{g}$, the last part of (b) follows from (a).

(c): Take $r \in Z(R)_{\text {fin }}$ and $g \in G$. By Proposition 11, we get that $\gamma_{g}\left(\operatorname{tr}_{\gamma}(r)\right)=\sum_{h \in G} \gamma_{g}\left(\gamma_{h}(r)\right)=$ $\sum_{h \in G} \gamma_{g h}(r) \epsilon_{g}=\operatorname{tr}_{\gamma}(r) \epsilon_{g}$. Therefore, $\operatorname{tr}_{\gamma}(r) \in Z(R)_{\text {fin }}^{\gamma}$. To prove the last statement, take $r \in Z(R)_{\mathrm{fin}}$ and $r^{\prime}, r^{\prime \prime} \in Z(R)_{\mathrm{fin}}^{\gamma}$. Then Proposition 12 implies that

$$
\begin{aligned}
\operatorname{tr}_{\gamma}\left(r^{\prime} r r^{\prime \prime}\right) & =\sum_{g \in G} \gamma_{g}\left(r^{\prime} r r^{\prime \prime}\right)=\sum_{g \in G} \gamma_{g}\left(r^{\prime}\right) \gamma_{g}(r) \gamma_{g}\left(r^{\prime \prime}\right)=\sum_{g \in G} r^{\prime} \epsilon_{g} \gamma_{g}(r) r^{\prime \prime} \epsilon_{g} \\
& =\sum_{g \in G} r^{\prime} \epsilon_{g} \gamma_{g}(r) \epsilon_{g} r^{\prime \prime}=\sum_{g \in G} r^{\prime} \gamma_{g}(r) r^{\prime \prime}=r^{\prime} \operatorname{tr}_{\gamma}(r) r^{\prime \prime} .
\end{aligned}
$$

(d): From the relation $r r^{-1}=1$, we get that $\gamma_{g}(r) \gamma_{g}\left(r^{-1}\right)=\epsilon_{g}$. Since $r \in Z(R)_{\text {fin }}^{\gamma}$, we get that $r \epsilon_{g} \gamma_{g}\left(r^{-1}\right)=\epsilon_{g}$. Thus, $r^{-1} r \gamma_{g}\left(r^{-1}\right)=r^{-1} \epsilon_{g}$. Hence, $\gamma_{g}\left(r^{-1}\right)=r^{-1} \epsilon_{g}$.

Corollary 18. Suppose that $\epsilon_{g}=0$, for all but finitely many $g \in G$. If $\operatorname{tr}_{\gamma}(1)$ is invertible in $R$, then $S / R$ is separable.

Proof. From Lemma 17(d), we get that $\operatorname{tr}_{\gamma}(1)^{-1} \in Z(R)_{\text {fin }}^{\gamma}$. Thus, by Lemma 17(c), it follows that $\operatorname{tr}_{\gamma}\left(\operatorname{tr}_{\gamma}(1)^{-1} 1\right)=\operatorname{tr}_{\gamma}(1)^{-1} \operatorname{tr}_{\gamma}(1)=1$. Hence, $S / R$ is separable due to Theorem 3 ,

Remark 19. The sufficient condition concerning invertibility of $\operatorname{tr}_{\gamma}(1)$ in Corollary 18$]$ is not necessary for separability (see Proposition [39).

\section{Semisimplicity, Hereditarity and Frobenius Properties}

In this section, we use Theorem 3 to find criteria for when epsilon-strongly graded rings are semisimple, hereditary and Frobenius (see Theorem 23 and Theorem 24). For the rest of this section, $S / R$ denotes a ring extension. Let res denote the restriction functor $S$-mod $\rightarrow R$ mod. The following two results are quite well-known, but, for the convenience of the reader, we have chosen to include the proofs.

Proposition 20. Let $S / R$ be separable and let $M$ be a left (right) $S$-module. If $\operatorname{res}(M)$ is left (right) projective, then $M$ is left (right) projective.

Proof. We only show the "left" part of the proof. The "right" part is shown in an analogous way and is therefore omitted. Take $n \in \mathbb{N}$ and $s_{j}, t_{j} \in S$, for $j \in\{1, \ldots, n\}$, such that $x=$ $\sum_{j=1}^{n} s_{j} \otimes t_{j}$ is a separability element of $S \otimes_{R} S$. Since $\operatorname{res}(M)$ is projective, $M$ has a dual $R$ basis $\left\{m_{i}\right\}_{i \in I}$ and $\left\{f_{i}\right\}_{i \in I}$. For each $i \in I$, define $F_{i}: M \rightarrow S$ by $F_{i}(m)=\sum_{j=1}^{n} s_{j} f_{i}\left(t_{j} m\right)$, for 
$m \in M$. We wish to show that $\left\{F_{i}\right\}_{i \in I}$ and $\left\{m_{i}\right\}_{i \in I}$ is a dual $S$-basis for $M$. First of all, clearly, each $F_{i}$ is additive. Next, for each $m \in M, \sum_{i \in I} F_{i}(m) m_{i}=\sum_{j=1}^{n} s_{j} \sum_{i \in I} f_{i}\left(t_{j} m\right) m_{i}=$ $\sum_{j=1}^{n} s_{j} t_{j} m=1 m=m$. Finally, take $i \in I, s \in S$ and $m \in M$. We need to show that $F_{i}(s m)=s F_{i}(m)$, or, in other words, that $\sum_{j=1}^{n} s_{j} f_{i}\left(t_{j} s m\right)=\sum_{j=1}^{n} s s_{j} f\left(t_{j} m\right)$. To see this, first notice that $x s=s x$ implies that $\sum_{j=1}^{n} s_{j} \otimes t_{j} s=\sum_{j=1}^{n} s s_{j} \otimes t_{j}$. From the left $S \otimes_{R} S$-module structure on $S \otimes_{R} M$ it follows that $\sum_{j=1}^{n} s_{j} \otimes t_{j} s m=\sum_{j=1}^{n} s s_{j} \otimes t_{j} m$. By applying the function $S \otimes_{R} M \ni a \otimes b \mapsto a \otimes f_{i}(b) \in S \otimes_{R} M$ to the last equality, we get that $\sum_{j=1}^{n} s_{j} \otimes f_{i}\left(t_{j} s m\right)=\sum_{j=1}^{n} s s_{j} \otimes f_{i}\left(t_{j} m\right)$. Finally, by applying the function $S \otimes_{R} M \ni$ $a \otimes b \mapsto a b \in M$ to the last equality, we get that $\sum_{j=1}^{n} s_{j} f_{i}\left(t_{j} s m\right)=\sum_{j=1}^{n} s s_{j} f_{i}\left(t_{j} m\right)$.

Lemma 21. $S$ is projective as a left $R$-module, if and only if, the functor res preserves projectives.

Proof. Suppose that $M$ is a projective left $S$-module. We wish to show that $M$, considered as a left $R$-module, is projective. Take a dual $S$-basis $\left\{m_{i}, f_{i}\right\}_{i \in I}$ for $M$ and a dual $R$-basis $\left\{s_{j}, g_{j}\right\}_{j \in J}$ for $S$. Then $\left\{s_{j} m_{i}, g_{j} \circ f_{i}\right\}_{(i, j) \in I \times J}$ is a dual basis of $M$ as a left $R$-module. Indeed, for $m \in M$ we have that

$$
\sum_{(i, j) \in I \times J}\left(g_{j} \circ f_{i}\right)(m)\left(s_{j} m_{i}\right)=\sum_{i}\left(\sum_{j} g_{j}\left(f_{i}(m)\right) s_{j}\right) m_{i}=\sum_{i} f_{i}(m) m_{i}=m .
$$

The converse is clear.

Recall that $R$ is called left (right) semisimple if all left (right) $R$-modules are semisimple. Notice that since $R$ is left semisimple if and only if $R$ is right semisimple, (see [13, Corollary (3.7)]) the left/right distinction is therefore unnecessary. Recall that $R$ is called left (right) hereditary if all submodules of left (right) projective modules over $R$ are again projective.

Corollary 22. Let $S / R$ be separable. If $R$ is semisimple (left/right hereditary and $S$ is projective as a left/right $R$-module), then $S$ is semisimple (left/right hereditary).

Proof. This follows from Proposition 20. Lemma 21 and the fact that a ring is semisimple (hereditary) if and only if every module (or submodule of a projective module) over the ring is projective (see [13, Theorem (2.8)]).

It is easy to see that if $S$ is a ring graded by a group $G$ and we put $R=S_{e}$, then semisimplicity (left/right hereditarity) of $R$ is always necessary for $S$ to be semisimple (left/right hereditary). In fact, this is true even in the more general setting of rings graded by categories (see [16, Proposition 3]). Now we determine sufficient conditions for semisimplicity (left/right hereditarity).

Theorem 23. Let $S$ be epsilon-strongly graded by a group $G$ and put $R=S_{e}$. Suppose that $R$ is semisimple (hereditary). If $1 \in \operatorname{tr}_{\gamma}\left(Z(R)_{\mathrm{fin}}\right.$ ) (and every $S_{g}$, for $g \in G$, is projective as a left/right $R$-module), then $S$ is semisimple (left/right hereditary). In particular, if $\epsilon_{g}=0$ for all but finitely many $g \in G$ and $\operatorname{tr}_{\gamma}(1)$ is invertible in $R$ (and every $S_{g}$, for $g \in G$, is projective as a left/right $R$-module), then $S$ is semisimple (left/right hereditary).

Proof. This follows from Theorem 3, Corollary 18, Corollary 22 and the fact that a direct sum of projective modules is projective. 
Recall that $S / R$ is called a Frobenius extension if there is a finite set $J, x_{j}, y_{j} \in S$, for $j \in J$, and an $R$-bimodule map $E: S \rightarrow R$ such that, for every $s \in S$, the equalities $s=\sum_{j \in J} x_{j} E\left(y_{j} s\right)=\sum_{j \in J}^{n} E\left(s x_{j}\right) y_{j}$ hold. In that case, $\left(E, x_{j}, y_{j}\right)$ is called a Frobenius system.

Theorem 24. If $S$ is epsilon-strongly graded by a finite group $G$ and we put $R=S_{e}$, then $S / R$ is a Frobenius extension.

Proof. Put $J=\left\{(g, i) \mid g \in G, 1 \leq i \leq n_{g}\right\}$, where $n_{g}$ is given by Definition 9. Since $G$ is finite, $J$ is finite. For each $j=(g, i) \in J$, define $x_{j}=u_{g}^{(i)}$ and $y_{j}=v_{g^{-1}}^{(i)}$. Define $E: S \rightarrow R$ by $E(s)=s_{e}$, for $s \in S$. Then, clearly, $E$ is an $R$-bimodule map. Take $s \in S$. Then

$$
\sum_{j \in J} x_{j} E\left(y_{j} s\right)=\sum_{g \in G} \sum_{i=1}^{n_{g}} u_{g}^{(i)} E\left(v_{g^{-1}}^{(i)} s\right)=\sum_{g \in G} \sum_{i=1}^{n_{g}} u_{g}^{(i)} v_{g^{-1}}^{(i)} s_{g}=\sum_{g \in G} \epsilon_{g} s_{g}=s
$$

and

$$
\sum_{j \in J}^{n} E\left(s x_{j}\right) y_{j}=\sum_{g \in G} \sum_{i=1}^{n_{g}} E\left(s u_{g}^{(i)}\right) v_{g^{-1}}^{(i)}=\sum_{g \in G} \sum_{i=1}^{n_{g}} s_{g^{-1}} u_{g}^{(i)} v_{g^{-1}}^{(i)}=\sum_{g \in G} s_{g^{-1}} \epsilon_{g}=s .
$$

Remark 25. The conclusion of Theorem 24 follows from Proposition 7 (iv). Indeed, since $G$ is finite, it follows that $S$ is finitely generated and projective as a left $R$-module. Using the notation used in Proposition 7, put $n=\oplus_{g \in G} n_{g}$. Then $n$ is an isomorphism of $R$-modules $S_{R} \rightarrow \operatorname{Hom}_{R}\left({ }_{R} S, R\right)_{R}$. Hence $S / R$ is a Frobenius extension, according to [12, Theorem 1.2].

Recall that if $T$ is a non-empty subset of $S$, then $C_{S}(T)$ denotes the set of $s \in S$ such that for every $t \in T$, the relation $s t=t s$ holds.

Proposition 26. Let $S / R$ be a Frobenius extension with Frobenius system $\left(E, x_{j}, y_{j}\right)$. Then $S / R$ is separable if and only if there is $d \in C_{S}(R)$ such that $\sum_{j \in J} x_{j} d y_{j}=1$.

Proof. See [12, Corollary 2.17].

Remark 27. Suppose that $S$ is epsilon-strongly graded by a finite group $G$ and put $R=S_{e}$. Using Theorem 24 and Proposition 26, we can, in this case, prove Theorem 3 in a different way. Indeed, using the above results, we can conclude that $S / R$ is separable if and only if there is $d \in C_{S}(R)$ such that $\sum_{g \in G} \gamma_{g}(d)=\sum_{g \in G} \sum_{i=1}^{n_{g}} u_{g}^{(i)} d v_{g^{-1}}^{(i)}=1$. Since $1 \in R$ it follows from the grading that $S / R$ is separable if and only if there is $c=d_{e} \in C_{R}(R)=Z(R)$ such that $\operatorname{tr}_{\gamma}(c)=1$.

\section{SimpliCity}

In this short section, we show that a result concerning simplicity for strongly graded rings from [17, Theorem 6.6] can be generalized to epsilon-strongly graded rings (see Proposition 29). Throughout this section, $S$ denotes an arbitrary unital ring which is epsilon-strongly graded by $G$ and we put $R=S_{e}$. Recall that $R$ is called a maximal commutative subring of $S$ if $C_{S}(R)=R$.

Lemma 28. If $I$ is a non-zero ideal of $S$, then $I \cap C_{S}(Z(R)) \neq\{0\}$. 
Proof. We claim that $S$ is right non-degenerate in the sense of [18, Definition 2]. If we assume that the claim holds, then the desired result follows from [18, Theorem 3]. Now we show the claim. Take $g \in G$ and any non-zero $s \in S_{g}$. Seeking a contradiction, suppose that $s S_{g^{-1}}=\{0\}$. Then $s S_{g^{-1}} S_{g}=\{0\}$. But since $\epsilon_{g^{-1}} \in S_{g^{-1}} S_{g}$ we get that $s=s \epsilon_{g^{-1}}=0$, which is a contradiction.

Recall that an ideal $I$ of $S$ is said to be graded if $I=\oplus_{g \in G}\left(I \cap S_{g}\right)$ holds. If $\{0\}$ and $S$ are the only graded ideals of $S$, then $S$ is said to be graded simple.

Proposition 29. If $R$ is a maximal commutative subring of $S$, then $S$ is simple if and only if $S$ is graded simple.

Proof. The "only if" statement is clear. Now we show the "if" statement. Let $I$ be a non-zero ideal of $S$. By the assumption we have $C_{S}(Z(R))=C_{S}(R)=R$. Hence, by Lemma 28 the set $J=I \cap C_{S}(Z(R))$ is a non-zero ideal of $R$. The set $S J S$ is a non-zero graded ideal of $S$ and thus, by graded simplicity of $S$, we get that $S=S J S=J$. This shows that $S$ is a simple ring.

\section{Partial Crossed Products}

In this section, we introduce epsilon-crossed products (see Definition 32). We show that the class of epsilon-crossed products coincides with the class of unital partial crossed products (see Theorem 33). Thereby, we obtain, in the special case of unital partial crossed products, a short proof of a more general result by Dokuchaev, Exel and Simón [8, Theorem 6.1] concerning when graded rings can be presented as partial crossed products. At the end of this section, we use Theorem 3 to reformulate Theorem 2 so that it holds for any, possibly infinite, group $G$ (see Theorem 35).

Definition 30. Let $S$ be a ring which is epsilon-strongly graded by $G$. Take $g \in G$ and $s \in S_{g}$. Then $s$ is called epsilon-invertible if there is $t \in S_{g^{-1}}$ such that $s t=\epsilon_{g}$ and $t s=\epsilon_{g^{-1}}$. We will refer to $t$ as the epsilon-inverse of $s$.

The usage of the term "the epsilon-inverse" is justified by the next result.

Proposition 31. Epsilon-inverses are unique.

Proof. Suppose that $g \in G, s \in S_{g}$ and $r, t \in S_{g^{-1}}$ satisfy the equalities $s t=s r=\epsilon_{g}$ and $t s=r s=\epsilon_{g^{-1}}$. Then $r=r \epsilon_{g}=r s t=\epsilon_{g^{-1}} t=t$.

Definition 32. Let $S$ be a ring which is epsilon-strongly graded by $G$. We say that $S$ is an epsilon-crossed product by $G$ if for each $g \in G$, there is an epsilon-invertible element in $S_{g}$.

Theorem 33. Let $S$ be a ring which is epsilon-strongly graded by $G$. Then $S$ is an epsiloncrossed product if and only if $S$ is a unital partial crossed product.

Proof. First we show the "only if" statement. Suppose that $S$ is an epsilon-crossed product. We will present $S$ as a unital partial crossed product. Take $g, h \in G$. Fix an epsilon-invertible element $s_{g} \in S_{g}$ with epsilon-inverse $t_{g^{-1}} \in S_{g^{-1}}$. We may assume that $s_{e}=t_{e}=1$. Put $D_{g}=S_{g} S_{g^{-1}}=R \epsilon_{g}, 1_{g}=\epsilon_{g}$ and $\delta_{g}=s_{g}$. Furthermore, define $\alpha_{g}: D_{g^{-1}} \rightarrow D_{g}$ by $\alpha_{g}\left(r \epsilon_{g^{-1}}\right)=s_{g} r t_{g^{-1}}$, for $r \in R$. Then $\alpha_{g}$ is well-defined. Indeed, if $r, r^{\prime} \in R$ satisfy $r \epsilon_{g^{-1}}=$ $r^{\prime} \epsilon_{g^{-1}}$, then $\alpha_{g}\left(r \epsilon_{g^{-1}}\right)=s_{g} r t_{g^{-1}}=s_{g} r \epsilon_{g^{-1}} t_{g^{-1}}=s_{g} r^{\prime} \epsilon_{g^{-1}} t_{g^{-1}}=s_{g} r^{\prime} t_{g^{-1}}=\alpha_{g}\left(r^{\prime} \epsilon_{g^{-1}}\right)$. The function $\alpha_{g}$ is bijective with inverse given by $\alpha_{g}^{-1}\left(r \epsilon_{g}\right)=t_{g^{-1}} r s_{g}$. Indeed, take $r \in R$. Then

$$
\alpha_{g}^{-1}\left(\alpha_{g}\left(r \epsilon_{g^{-1}}\right)\right)=t_{g^{-1}} s_{g} r t_{g^{-1}} s_{g}=\epsilon_{g^{-1}} r \epsilon_{g^{-1}}=r \epsilon_{g^{-1}}
$$


and

$$
\alpha_{g}\left(\alpha_{g}^{-1}\left(r \epsilon_{g}\right)\right)=s_{g} t_{g^{-1}} r s_{g} t_{g^{-1}}=\epsilon_{g} r \epsilon_{g}=r \epsilon_{g} .
$$

The function $\alpha_{g}$ is clearly additive. Also $\alpha_{g}\left(\epsilon_{g^{-1}}\right)=s_{g} \epsilon_{g^{-1}} t_{g^{-1}}=s_{g} t_{g^{-1}}=\epsilon_{g}$. Now we show that $\alpha_{g}$ is multiplicative. Take $r, r^{\prime} \in R$. Then

$$
\alpha_{g}\left(r r^{\prime} \epsilon_{g}\right)=s_{g} r r^{\prime} t_{g^{-1}}=s_{g} r \epsilon_{g^{-1}} r^{\prime} t_{g-1}=s_{g} r t_{g^{-1}} s_{g} r^{\prime} t_{g^{-1}}=\alpha_{g}\left(r \epsilon_{g^{-1}}\right) \alpha_{g}\left(r^{\prime} \epsilon_{g^{-1}}\right) .
$$

Next put $w_{g, h}=s_{g} s_{h} t_{(g h)^{-1}}$. Since $w_{g, h} \in R, \epsilon_{g} s_{g}=s_{g}$ and $t_{(g h)^{-1}} \epsilon_{g h}=t_{(g h)^{-1}}$, it follows that $w_{g, h} \in D_{g} D_{g h}$. Now we show that $w_{g, h}$ is a unit in $D_{g} D_{g h}$. To this end, first notice that $\alpha_{g}\left(\epsilon_{g^{-1}} \epsilon_{h}\right)=\epsilon_{g} \epsilon_{g h}$. In fact, from (P2) (see below), we get that there is $r \in D_{g^{-1}} D_{h}$ such that $\alpha_{g}(r)=\epsilon_{g} \epsilon_{g h}$. Since $\epsilon_{g} \epsilon_{g h}$ is the identity of $D_{g} D_{g h}$, we get that $\alpha_{g}\left(\epsilon_{g^{-1}} \epsilon_{h}\right)=\alpha_{g}\left(\epsilon_{g^{-1}} \epsilon_{h}\right) \epsilon_{g} \epsilon_{g h}=\alpha_{g}\left(\epsilon_{g^{-1}} \epsilon_{h}\right) \alpha_{g}(r)=\alpha_{g}\left(\epsilon_{g^{-1}} \epsilon_{h} r\right)=\alpha_{g}(r)=\epsilon_{g} \epsilon_{g h}$. Put $v_{g, h}=s_{g h} t_{h^{-1}} t_{g^{-1}} \epsilon_{g} \epsilon_{g h}$. Then $v_{g, h} \in D_{g} D_{g h}$ and

$$
\begin{aligned}
w_{g, h} v_{g, h} & =s_{g} s_{h} t_{(g h)^{-1}} s_{g h} t_{h^{-1}} t_{g^{-1}}=s_{g} s_{h} \epsilon_{(g h)^{-1}} t_{h^{-1}} t_{g^{-1}}=s_{g} s_{h} t_{h^{-1}} t_{g^{-1}} \\
& =s_{g} \epsilon_{h} t_{g^{-1}}=s_{g} \epsilon_{h} \epsilon_{g^{-1}} t_{g^{-1}}=\alpha_{g}\left(\epsilon_{h} \epsilon_{g^{-1}}\right)=\epsilon_{g} \epsilon_{g h}
\end{aligned}
$$

and

$$
\begin{aligned}
v_{g, h} w_{g, h} & =s_{g h} t_{h^{-1}} t_{g^{-1}} s_{g} s_{h} t_{(g h)^{-1}}=s_{g h} t_{h^{-1}} \epsilon_{g^{-1}} s_{h} t_{(g h)^{-1}}=s_{g h} t_{h^{-1}} s_{h} t_{(g h)^{-1}} \\
& =s_{g h} \epsilon_{h^{-1}} t_{(g h)^{-1}}=s_{g h} \epsilon_{h^{-1}} \epsilon_{(g h)^{-1}} t_{(g h)^{-1}}=\alpha_{g h}\left(\epsilon_{(g h)^{-1}} \epsilon_{h^{-1}}\right)=\epsilon_{g h} \epsilon_{g} \epsilon_{g h} \\
& =\epsilon_{g h} \epsilon_{g} .
\end{aligned}
$$

Now we check conditions (P1)-(P6) from the introduction.

(P1): Using that $\epsilon_{e}=1$, we get that $D_{e}=R$. Since $\gamma_{e}=\mathrm{id}{ }_{R}$, we get that $\alpha_{e}=\mathrm{id}{ }_{R}$.

(P2): First notice that $\alpha_{g}\left(D_{g^{-1}} D_{h}\right)=s_{g} D_{g^{-1}} D_{h} t_{g^{-1}}=s_{g} S_{g^{-1}} S_{g} S_{h} S_{h^{-1}} t_{g^{-1}}$. Since $s_{g} \in S_{g}$, and thereby $s_{g} S_{g^{-1}} \in R$, we can conclude that

$$
\begin{aligned}
\alpha_{g}\left(D_{g^{-1}} D_{h}\right) & =\epsilon_{g} s_{g} S_{g^{-1}} \epsilon_{g h} S_{g} S_{h} S_{h^{-1}} t_{g^{-1}}=\epsilon_{g} \epsilon_{g h}\left(s_{g} S_{g^{-1}} S_{g} S_{h} S_{h^{-1}} t_{g^{-1}}\right) \\
& \subseteq D_{g} D_{g h} R=D_{g} D_{g h} .
\end{aligned}
$$

Now we show the reversed inclusion. Take $r \in R$. Put $r^{\prime}=t_{g^{-1}} r \epsilon_{g} \epsilon_{g h} s_{g} \in R$. Then $\epsilon_{g^{-1}} r^{\prime}=r^{\prime}$. Also, since $\epsilon_{g h} s_{g} \in S_{g h} S_{(g h)^{-1}} s_{g} \subseteq S_{g h} S_{h^{-1}}$, it follows that $r^{\prime} \epsilon_{h}=r^{\prime}$. Thus, $r^{\prime} \in D_{g^{-1}} D_{h}$. Now,

$$
\alpha_{g}\left(r^{\prime}\right)=s_{g} t_{g^{-1}} r \epsilon_{g} \epsilon_{g h} s_{g} t_{g^{-1}}=\epsilon_{g} r \epsilon_{g} \epsilon_{g h} \epsilon_{g}=r \epsilon_{g} \epsilon_{g h} .
$$

(P3): Take $r \in D_{h^{-1}} D_{(g h)^{-1}}$. Then

$$
\alpha_{g}\left(\alpha_{h}(r)\right) w_{g, h}=s_{g} s_{h} r t_{h^{-1}} t_{g^{-1}} s_{g} s_{h} t_{(g h)^{-1}}=s_{g} s_{h} r t_{h^{-1}} \epsilon_{g^{-1}} s_{h} t_{(g h)^{-1}},
$$

and the last expression equals

$$
\begin{gathered}
s_{g} s_{h} r t_{h^{-1}} s_{h} t_{(g h)^{-1}}=s_{g}\left(s_{h} r\right) \epsilon_{h^{-1}} t_{(g h)^{-1}}=s_{g} s_{h} r t_{(g h)^{-1}}=s_{g} s_{h} r \epsilon_{(g h)^{-1}} t_{(g h)^{-1}} \\
=\left(s_{g} s_{h} t_{(g h)^{-1}}\right) s_{g h} r t_{(g h)^{-1}}=w_{g, h} s_{g h} r t_{(g h)^{-1}}=w_{g, h} \alpha_{g h}(r) .
\end{gathered}
$$

(P4): Using that $s_{e}=1$, we get that $w_{g, e}=s_{g} s_{e} t_{g^{-1}}=s_{g} t_{g^{-1}}=\epsilon_{g}$ and $w_{e, g}=s_{e} s_{g} t_{g^{-1}}=$ $s_{g} t_{g^{-1}}=\epsilon_{g}$.

(P6): Notice first that $S_{g}=D_{g} \delta_{g}$. In fact, since $s_{g} \in S_{g}$ and $D_{g} \subseteq R$ it follows that $S_{g} \supseteq$ $D_{g} s_{g}$. On the other hand, take $s_{g}^{\prime} \in S_{g}$. Then $s_{g}^{\prime}=s_{g}^{\prime} \epsilon_{g^{-1}}=s_{g}^{\prime} t_{g^{-1}} s_{g}=s_{g}^{\prime} t_{g^{-1}} \epsilon_{g} s_{g} \in D_{g} s_{g}$. Thus $S_{g} \subseteq D_{g} s_{g}$. So we get that $S=\oplus_{g \in G} D_{g} s_{g}$. Also, if $s=\sum_{g \in G} r_{g} s_{g}$, for $r_{g} \in D_{g}$, then the $r_{g}$ 's are unique. Indeed, suppose that $r_{g} s_{g}=r_{g}^{\prime} s_{g}$ for some $r_{g}, r_{g}^{\prime} \in D_{g}$. Using that $\epsilon_{g}$ 
is the multiplicative identity element of $D_{g}=S_{g} S_{g^{-1}}$, we get that $r_{g}=r_{g} \epsilon_{g}=r_{g} s_{g} t_{g^{-1}}=$ $r_{g}^{\prime} s_{g} t_{g^{-1}}=r_{g}^{\prime} \epsilon_{g}=r_{g}^{\prime}$. Take $r \in D_{g}$ and $r^{\prime} \in D_{h}$. Then

$$
\begin{aligned}
\left(r s_{g}\right)\left(r^{\prime} s_{h}\right) & =r s_{g} \epsilon_{g^{-1}} r^{\prime} \epsilon_{g^{-1}} s_{h}=r s_{g} \epsilon_{g^{-1}} r^{\prime} t_{g^{-1}} s_{g} s_{h}=r \alpha_{g}\left(\epsilon_{g^{-1}} r^{\prime}\right) s_{g} s_{h} \\
& =r \alpha_{g}\left(\epsilon_{g^{-1}} r^{\prime}\right) s_{g} s_{h} \epsilon_{(g h)^{-1}}=r \alpha_{g}\left(\epsilon_{g^{-1}} r^{\prime}\right) s_{g} s_{h} t_{(g h)^{-1}} s_{g h} \\
& =r \alpha_{g}\left(\epsilon_{g^{-1}} r^{\prime}\right) w_{g, h} s_{g h} .
\end{aligned}
$$

(P5): Take $r \in D_{g^{-1}} D_{h} D_{h l}$. Then

$$
\left(s_{g} r s_{h}\right) s_{l}=\left(\alpha_{g}(r) s_{g} s_{h}\right) s_{l}=\left(\alpha_{g}(r) w_{g, h} s_{g h}\right) s_{l}=\alpha_{g}(r) w_{g, h} w_{g h, l} s_{g h l}
$$

and

$$
s_{g}\left(r s_{h} s_{l}\right)=s_{g}\left(r w_{h, l} s_{h l}\right)=\alpha_{g}\left(r w_{h, l}\right) w_{g, h l} s_{g h l} .
$$

The claim now follows from the proof of (P6) and associativity.

Now we show the "if" statement. Suppose that $S=\oplus_{g \in G} D_{g} \delta_{g}$ is a unital partial crossed product. Take $g \in G$. Since $S_{g} S_{g^{-1}}=D_{g} \delta_{e}$, we can put $\epsilon_{g}=1_{g} \delta_{e}$. What remains to show is associativity of $S$. This has already been shown in a more general context (see [8, Theorem 2.4]). Here we provide a short direct proof for unital twisted partial actions. To this end, take $g, h, l \in G, a \in D_{g}, b \in D_{h}$ and $c \in D_{l}$. Then

$$
\left(a \delta_{g} b \delta_{h}\right) c \delta_{l}=\left(a \alpha_{g}\left(1_{g^{-1}} b\right) w_{g, h} \delta_{g h}\right) c \delta_{l}=a \alpha_{g}\left(1_{g^{-1}} b\right) w_{g, h} \alpha_{g h}\left(1_{(g h)^{-1}} c\right) w_{g h, l} \delta_{g h l} .
$$

By (P2), the last expression equals

$$
a \alpha_{g}\left(1_{g^{-1}} b\right) w_{g, h} \alpha_{g h}\left(1_{h^{-1}} 1_{(g h)^{-1}} c\right) w_{g h, l} \delta_{g h l},
$$

which, in turn, by (P3), equals

$$
\begin{aligned}
& a \alpha_{g}\left(1_{g^{-1}} b\right) \alpha_{g}\left(\alpha_{h}\left(1_{h^{-1}} 1_{(g h)^{-1}} c\right)\right) w_{g, h} w_{g h, l} \delta_{g h l}= \\
& a \alpha_{g}\left(1_{g^{-1}} b \alpha_{h}\left(1_{h^{-1}} 1_{(g h)^{-1}} c\right)\right) w_{g, h} w_{g h, l} \delta_{g h l}=a \alpha_{g}\left(1_{g^{-1}} b \alpha_{h}\left(1_{h^{-1}} c\right)\right) w_{g, h} w_{g h, l} \delta_{g h l} .
\end{aligned}
$$

By (P5), this equals

$$
a \alpha_{g}\left(1_{g^{-1}} b \alpha_{h}\left(1_{h^{-1}}\right) w_{h, l}\right) w_{g, h l} \delta_{g h l}=a \delta_{g}\left(b \alpha_{h}\left(1_{h^{-1}} c\right) w_{h, l} \delta_{h l}\right)=a \delta_{g}\left(b \delta_{h} c \delta_{l}\right) .
$$

Definition 34. Let $S=R \star_{\alpha}^{w} G$ be a unital partial crossed product, and let $Z(R)_{\alpha \text {,fin }}$ denote the set of $r \in Z(R)$ with the property that for all but finitely many $g \in G$, the relation $r 1_{g}=0$ holds. Define the trace map $t_{\alpha}: Z(R)_{\alpha, \text { fin }} \rightarrow Z(R)$ by $t_{\alpha}(r)=\sum_{g \in G} \alpha_{g}\left(r 1_{g^{-1}}\right)$, for $r \in Z(R)_{\alpha, \text { fin }}$.

Theorem 35. If $S$ is a unital partial crossed product of a group $G$ over $R$, then $S / R$ is separable if and only if $1 \in \operatorname{tr}_{\alpha}\left(Z(R)_{\mathrm{fin}}\right)$.

Proof. This follows from Theorem 3 and Theorem 33 ,

Lemma 36. Let $S$ be a unital partial crossed product of a group $G$ over $R$ and take $g \in G$. If $D_{g}$ is projective as a left (right) $R$-module, then $S_{g}=D_{g} \delta_{g}$ is projective as a left (right) $R$-module.

Proof. The "left" part is trivial since the left action of $R$ on $S_{g}$ is defined by the left action of $R$ on $D_{g}$. Now we show the "right" part. Suppose that $D_{g}$ is projective as a right $R$-module. Let $\left\{d_{i}, f_{i}\right\}_{i \in I}$ be a dual basis for $D_{g}$ as a right $R$-module. For each $i \in I$, define $\bar{f}_{i}: D_{g} \rightarrow R$ 
by the relations $\bar{f}_{i}(d)=\alpha_{g}^{-1}\left(1_{g} f_{i}(d)\right)$, for $d \in D_{g}$. Then $\left\{d_{i} \delta_{g}, \bar{f}_{i}\right\}_{i \in I}$ is a dual basis for $S_{g}=D_{g} \delta_{g}$ as a right $R$-module. In fact, if $d \in D_{g}$, then

$$
\sum_{i \in I}\left(d_{i} \delta_{g}\right)\left(\bar{f}_{i}(d) \delta_{e}\right)=\sum_{i \in I} d_{i} \alpha_{g}\left(\bar{f}_{i}(d)\right) w_{g, e} \delta_{g}=\sum_{i \in I} d_{i} 1_{g} f_{i}(d) \delta_{g}=d \delta_{g}
$$

Theorem 37. Let $S$ be a unital partial crossed product of a group $G$ over $R$, and let $R$ is semisimple (left/right hereditary). If $1 \in \operatorname{tr}_{\gamma}\left(Z(R)_{\mathrm{fin}}\right)$, then $S$ is semisimple (left/right hereditary). In particular, if $\epsilon_{g}=0$ for all but finitely many $g \in G$ and $\operatorname{tr}_{\gamma}(1)$ is invertible in $R$, then $S$ is semisimple (left/right hereditary).

Proof. The "semisimple" part follows from Theorem 23, The "hereditary" part follows from Theorem 23. Lemma 36 and the fact that all the ideals $D_{g}$, for $g \in G$, of the hereditary ring $R$, are left/right projective.

\section{Examples: A Dade-Like Construction}

In this section, we provide a class of examples of separable epsilon-strongly graded rings, neither of which are strongly graded, nor partial crossed products, in any natural way. Our inspiration comes from the first known example (due to E. Dade, according to 6 , Example 2.9]) of a strongly graded ring which is not a crossed product. Namely, suppose that $A$ is a commutative unital ring with a non-zero multiplicative identity $1_{A}$. Put

$$
S=M_{3}(A), \quad R=\left(\begin{array}{ccc}
A & A & 0 \\
A & A & 0 \\
0 & 0 & A
\end{array}\right) \text { and } T=\left(\begin{array}{ccc}
0 & 0 & A \\
0 & 0 & A \\
A & A & 0
\end{array}\right) .
$$

Then $S$ is strongly $\mathbb{Z}_{2}$-graded with $S_{0}=R$ and $S_{1}=T$, but $S$ is not a crossed product of $\mathbb{Z}_{2}$ over $R$ since $T$ does not contain any element which is invertible in $S$. Our idea is to postulate another unital commutative ring $B$ with a non-zero multiplicative identity $1_{B}$ such that $B$ is an ideal of $A$ with $B \subsetneq A$. Now we modify Dade's example by putting

$$
S=\left(\begin{array}{ccc}
A & A & B \\
A & A & B \\
B & B & A
\end{array}\right), \quad R=\left(\begin{array}{ccc}
A & A & 0 \\
A & A & 0 \\
0 & 0 & A
\end{array}\right) \text { and } T=\left(\begin{array}{ccc}
0 & 0 & B \\
0 & 0 & B \\
B & B & 0
\end{array}\right)
$$

Proposition 38. The ring $S$ is epsilon-strongly $\mathbb{Z}_{2}$-graded with $S_{0}=R$ and $S_{1}=T$. With this grading, $S$ is neither strongly graded, nor a partial crossed product. Moreover, the ring extension $S / R$ is separable.

Proof. If we put

$$
\epsilon_{0}=\left(\begin{array}{ccc}
1_{A} & 0 & 0 \\
0 & 1_{A} & 0 \\
0 & 0 & 1_{A}
\end{array}\right) \quad \text { and } \quad \epsilon_{1}=\left(\begin{array}{ccc}
1_{B} & 0 & 0 \\
0 & 1_{B} & 0 \\
0 & 0 & 1_{B}
\end{array}\right)
$$

then it is clear that $R R=R \epsilon_{0}$ and $T T=\epsilon_{1} R$. Hence $S$ is epsilon-strongly $\mathbb{Z}_{2}$-graded. The equality $T T=\epsilon_{1} R$ also shows that $S$ is not strongly graded, since $1_{A} \notin B$. Seeking a contradiction, suppose that this grading presents $S$ as a partial crossed product of $\mathbb{Z}_{2}$ over 
$R$. Then there is $\delta_{1} \in T$ and a non-zero unital ideal $D$ of $R$ such that $T=D \delta_{1}$. Take unital ideals $I$ and $J$ of $A$, of which at least one is non-zero, such that

$$
D=\left(\begin{array}{lll}
I & I & 0 \\
I & I & 0 \\
0 & 0 & J
\end{array}\right)
$$

take $b_{1}, b_{2}, b_{3}, b_{4} \in B$ such that

$$
\delta_{1}=\left(\begin{array}{ccc}
0 & 0 & b_{3} \\
0 & 0 & b_{4} \\
b_{1} & b_{2} & 0
\end{array}\right)
$$

and take $w_{11}, w_{12}, w_{21}, w_{22} \in I$ and $w_{33} \in J$ such that

$$
w_{1,1}^{-1}=\left(\begin{array}{ccc}
w_{11} & w_{12} & 0 \\
w_{21} & w_{22} & 0 \\
0 & 0 & w_{33}
\end{array}\right) \text {. }
$$

From (P6) it follows that

$$
\left(w_{1,1}^{-1} \delta_{1}\right)\left(1_{D} \delta_{1}\right)=1_{D} \delta_{0} .
$$

By a straightforward calculation, (11) can be rewritten as

$$
\left(\begin{array}{ccc}
1_{J} b_{1}\left(w_{11} b_{3}+w_{12} b_{4}\right) & 1_{J} b_{2}\left(w_{11} b_{3}+w_{12} b_{4}\right) & 0 \\
1_{J} b_{1}\left(w_{21} b_{3}+w_{22} b_{4}\right) & 1_{J} b_{2}\left(w_{21} b_{3}+w_{22} b_{4}\right) & 0 \\
0 & 0 & 1_{I} w_{33}\left(b_{1} b_{3}+b_{2} b_{4}\right)
\end{array}\right)=\left(\begin{array}{ccc}
1_{I} & 0 & 0 \\
0 & 1_{I} & 0 \\
0 & 0 & 1_{J}
\end{array}\right) .
$$

From (2) it follows in particular that

$$
1_{J} b_{1}\left(w_{11} b_{3}+w_{12} b_{4}\right)=1_{I} \quad \text { and } \quad 1_{I} w_{33}\left(b_{1} b_{3}+b_{2} b_{4}\right)=1_{J} .
$$

Thus, $1_{I} \in J$ and $1_{J} \in I$. Hence $I=J$ and so we get that $1_{I}=1_{J} \neq 0$. By a straightforward calculation the determinant of the left hand side of (2) is zero. This contradicts the fact that the determinant of the right hand side of (2) equals $1_{I} \neq 0$.

Now we show that $S / R$ is separable. First of all, it is easy to show that

$$
Z(R)=\left\{\left(\begin{array}{ccc}
a & 0 & 0 \\
0 & a & 0 \\
0 & 0 & a^{\prime}
\end{array}\right) \mid a, a^{\prime} \in A\right\} .
$$

We know that $\gamma_{0}: Z(R) \rightarrow Z(R)$ is the identity map on $Z(R)$. Now we determine $\gamma_{1}: Z(R) \rightarrow$ $Z(R)$. To this end, let $e_{i j}$ denote the $3 \times 3$ matrix over $A$ with $1_{A}$ in the $i j$ th position, and zeros elsewhere. Since

$$
1_{B} e_{13} 1_{B} e_{31}+1_{B} e_{23} 1_{B} e_{32}+1_{B} e_{31} 1_{B} e_{13}=\epsilon_{1}
$$

the map $\gamma_{1}: Z(R) \rightarrow Z(R)$ is defined by

$$
Z(R) \ni r \mapsto 1_{B} e_{13} r 1_{B} e_{31}+1_{B} e_{23} r 1_{B} e_{32}+1_{B} e_{31} r 1_{B} e_{13}
$$

Thus, the trace map $\operatorname{tr}_{\gamma}: Z(R) \rightarrow Z(R)$ is defined by

$$
Z(R) \ni r \mapsto r+1_{B} e_{13} r 1_{B} e_{31}+1_{B} e_{23} r 1_{B} e_{32}+1_{B} e_{31} r 1_{B} e_{13} .
$$

By Theorem 3, we can deduce that $S / R$ is separable if we can find

$$
r=\left(\begin{array}{ccc}
a & 0 & 0 \\
0 & a & 0 \\
0 & 0 & a^{\prime}
\end{array}\right) \in Z(R)
$$


such that

$$
\operatorname{tr}_{\gamma}(r)=\left(\begin{array}{ccc}
1_{A} & 0 & 0 \\
0 & 1_{A} & 0 \\
0 & 0 & 1_{A}
\end{array}\right)
$$

By a straightforward calculation, the last relation is equivalent to the set of equations $a+$ $1_{B} a^{\prime}=1_{A}$ and $a^{\prime}+1_{B} a=1_{A}$. It is easy to see that this set of equations is satisfied if we e.g. put $a=1_{A}$ and $a^{\prime}=1_{A}-1_{B}$. Therefore, $S / R$ is separable.

It is easy to give concrete examples of rings $A$ and $B$ which fit into the above construction. In fact, from now on in this section, suppose that $\mathbb{F}$ is a field, $A=\mathbb{F} \times \mathbb{F}$ and $B=\mathbb{F} \times\{0\}$. In that case, the sufficient condition for separability in Corollary 18 is not necessary.

Proposition 39. With the above notation, $\operatorname{tr}_{\gamma}\left(1_{R}\right)$ is invertible in $R$ if and only if $\operatorname{char}(\mathbb{F}) \neq$ 2.

Proof. Since

$$
1_{R}=\left(\begin{array}{ccc}
1_{A} & 0 & 0 \\
0 & 1_{A} & 0 \\
0 & 0 & 1_{A}
\end{array}\right)
$$

we get, from the proof of Proposition 38, that

$$
\operatorname{tr}_{\gamma}\left(1_{R}\right)=\left(\begin{array}{ccc}
1_{A}+1_{B} & 0 & 0 \\
0 & 1_{A}+1_{B} & 0 \\
0 & 0 & 1_{A}+1_{B}
\end{array}\right) .
$$

Since $1_{A}+1_{B}=(1,1)+(1,0)=(2,1)$, we get that $\operatorname{tr}_{\gamma}\left(1_{R}\right)$ is invertible in $R$ if and only if $\operatorname{char}(\mathbb{F}) \neq 2$.

Remark 40. In [14, Remark II.5.1.6] the authors write that "If $S$ is an arbitrary graded ring by a finite group $G$ we do not know whether separability of $S$ over $S_{e}$ implies that $S$ is strongly graded. This seems very likely however." Proposition 38 is a counterexample to this assumption. Moreover, it is the first known example for which all the homogeneous components of the grading are non-zero. Using the same method as in [1, Remark 3.2], one may construct a counterexample with a trivial grading, i.e. with $S=S_{e}$.

\section{EXAmples: Morita RINGS}

Let $\left(A, B,{ }_{A} M_{B}, B N_{A}, \varphi, \phi\right)$ be a strict Morita context. It consists of unital rings $A$ and $B$, an $A-B$-bimodule $M$, an $B-A$-bimodule $N$, an $A-A$-bimodule epimorphism $\varphi: M \otimes_{B} N \rightarrow$ $A$ and an $B-B$-bimodule epimorphism $\phi: N \otimes_{A} M \rightarrow B$.

The associated Morita ring is the set

$$
S=\left(\begin{array}{cc}
A & M \\
N & B
\end{array}\right)
$$

equipped with the natural addition and with a multiplication defined by

$$
\left(\begin{array}{cc}
a_{1} & m_{1} \\
n_{1} & b_{1}
\end{array}\right) *\left(\begin{array}{cc}
a_{2} & m_{2} \\
n_{2} & b_{2}
\end{array}\right)=\left(\begin{array}{cc}
a_{1} a_{2}+\varphi\left(m_{1} \otimes n_{2}\right) & a_{1} m_{2}+m_{1} b_{2} \\
n_{1} a_{2}+b_{1} n_{2} & \phi\left(n_{1} \otimes m_{2}\right)+b_{1} b_{2}
\end{array}\right)
$$

for $a_{1}, a_{2} \in A, b_{1}, b_{2} \in B, m_{1}, m_{2} \in M$ and $n_{1}, n_{2} \in N$. Let $G$ be an infinite cyclic group, generated by $g$. We can define a $G$-grading on $S$ by putting

$$
R=S_{e}=\left(\begin{array}{cc}
A & 0 \\
0 & B
\end{array}\right), \quad S_{g}=\left(\begin{array}{cc}
0 & M \\
0 & 0
\end{array}\right), \quad S_{g^{-1}}=\left(\begin{array}{cc}
0 & 0 \\
N & 0
\end{array}\right)
$$


and $S_{h}=\left\{\left(\begin{array}{ll}0 & 0 \\ 0 & 0\end{array}\right)\right\}$ for every $h \in G \backslash\left\{e, g, g^{-1}\right\}$. It is easy to see that

$$
S_{g} S_{g^{-1}}=\left(\begin{array}{cc}
\operatorname{Im}(\varphi) & 0 \\
0 & 0
\end{array}\right)=\left(\begin{array}{cc}
A & 0 \\
0 & 0
\end{array}\right)
$$

and

$$
S_{g^{-1}} S_{g}=\left(\begin{array}{cc}
0 & 0 \\
0 & \operatorname{Im}(\phi)
\end{array}\right)=\left(\begin{array}{cc}
0 & 0 \\
0 & B
\end{array}\right)
$$

and thus $S$ is obviously not strongly graded. However, $S$ is epsilon-strongly graded. Indeed, if we put

$$
\epsilon_{g}=\left(\begin{array}{cc}
1_{A} & 0 \\
0 & 0
\end{array}\right), \quad \epsilon_{g^{-1}}=\left(\begin{array}{cc}
0 & 0 \\
0 & 1_{B}
\end{array}\right) \text { and } \epsilon_{e}=\epsilon_{g}+\epsilon_{g^{-1}}
$$

then it is easy to verify that this yields an epsilon-strong $G$-grading on $S$. From the fact that $\operatorname{Supp}(S)=\left\{g \in G \mid S_{g} \neq\{0\}\right\}$ is finite, we immediately see that

$$
Z(R)_{\text {fin }}=Z(R)=\left(\begin{array}{cc}
Z(A) & 0 \\
0 & Z(B)
\end{array}\right) .
$$

Remark 41. With this grading one can find examples in which the Morita ring $S$ is not a partial crossed product of $G$ over $R=S_{e}$. Indeed, let $P$ be a progenerator in the category $\bmod -R$, of right $R$-modules. It follows by [11, Theorem 3.20] that $\left(\operatorname{End} P_{R}, R, P, P^{*}=\right.$ $\left.\operatorname{hom}\left(P_{R}, R\right), \varphi, \phi\right)$, where $\varphi: P^{*} \otimes_{\operatorname{End} P_{R}} P \ni f \otimes p \rightarrow f(p) \in R$ and $\varphi: P \otimes_{R} P^{*} \ni p \otimes f \rightarrow$ $f_{p} \in \operatorname{End} P_{R}$, and $f_{p}(r)=p f(r)$, for all $r \in R$, is a strict Morita context. Consider the

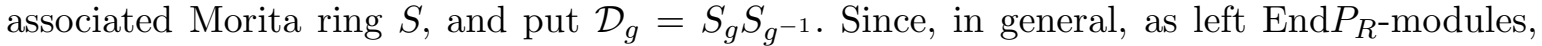
End $P_{R}$ is not isomorphic to $P$, it follows by [8, Theorem 6.5] that $S$ is not a partial crossed product of $G$ over $S_{e}$.

A concrete example is obtained by taking a commutative unital ring $R$ and $P=R^{n}$, for some $n>1$.

Proposition 42. Let $\left(A, B,{ }_{A} M_{B},{ }_{B} N_{A}, \varphi, \phi\right)$ be a strict Morita context, and let $S$ be the associated Morita ring. Then the extension $S / R$ is separable.

Proof. We know that $\gamma_{0}: Z(R) \rightarrow Z(R)$ is the identity map on $Z(R)$. Now we determine $\gamma_{g}$ and $\gamma_{g^{-1}}$. Let $\sum_{i} m_{i} \otimes n_{i} \in M \otimes_{B} N$ be such that $\sum_{i} \varphi\left(m_{i} \otimes n_{i}\right)=1_{A}$ and $\sum_{j} n_{j} \otimes m_{j} \in N \otimes_{A} M$ with $\sum_{j} \phi\left(n_{j} \otimes m_{j}\right)=1_{B}$. Then for $a e_{11}+b e_{22} \in Z(R)$ one has that

$$
\gamma_{g}\left(a e_{11}+b e_{22}\right)=\sum_{i} \varphi\left(m_{i} b \otimes n_{i}\right) e_{11}
$$

and

$$
\gamma_{g^{-1}}\left(a e_{11}+b e_{22}\right)=\sum_{j} \phi\left(n_{j} a \otimes m_{j}\right) e_{22} .
$$

Then the trace map $\operatorname{tr}_{\gamma}: Z(R) \rightarrow Z(R)$ is given by

$$
a e_{11}+b e_{22} \mapsto a e_{11}+b e_{22}+\sum_{i} \varphi\left(m_{i} b \otimes n_{i}\right) e_{11}+\sum_{j} \phi\left(n_{j} a \otimes m_{j}\right) e_{22} .
$$

From this it follows that $\operatorname{tr}\left(1_{A} e_{11}\right)=1_{A} e_{11}+1_{B} e_{22}$, and hence $S / R$ is separable due to Theorem 3 , 
Example 43. Let $T=\oplus_{g \in G} T_{g}$ be a ring which is strongly graded by a group $G$. Fix $g \in G$ and consider the strict Morita context $\left(T_{e}, T_{e}, T_{g}, T_{g^{-1}}, \varphi, \phi\right)$ where $\varphi: T_{g} \otimes_{T_{e}} T_{g^{-1}} \rightarrow T_{e}$ and $\phi: T_{g^{-1}} \otimes_{T_{e}} T_{g} \rightarrow T_{e}$ are the canonical $T_{e}$-bimodule isomorphisms (see [22, Corollary 3.1.2] and [5]). The corresponding Morita $\operatorname{ring} S=\left(\begin{array}{cc}T_{e} & T_{g} \\ T_{g}-1 & T_{e}\end{array}\right)$ is epsilon-strongly graded by an infinite cyclic group $G$, generated by $g$, as described above. By Proposition $42, S$ is separable over $R=\left(\begin{array}{cc}T_{e} & 0 \\ 0 & T_{e}\end{array}\right)$.

Remark 44. If $S$ is a ring which is strongly graded by $G$, then $G=\operatorname{Supp}(S)=\{g \in G \mid$ $\left.S_{g} \neq\{0\}\right\}$ necessarily holds. However, if $S$ is only epsilon-strongly graded by $G$, then $\operatorname{Supp}(S)$ need not even be a subgroup of $G$. Indeed, consider Example 43 and notice that $g$ belongs to $\operatorname{Supp}(S)$ but that $\left.S_{g^{2}}=\left\{\begin{array}{ll}0 & 0 \\ 0 & 0\end{array}\right)\right\}$. Hence, in this case $\operatorname{Supp}(S)$ is not closed under group multiplication.

\section{ACKNOWLEDGEMENT}

The authors are grateful to Ruy Exel for having pointed out the equivalence between (ii) and (iii) in Proposition 7.

\section{REFERENCES}

[1] D. Bagio, J. Lazzarin and A. Paques, Crossed Products by Twisted Partial Acctions: Separability, Semisimplicity and Frobenius Properties, Comm. Algebra 38(2), 496-508 (2010).

[2] S. Caenepeel and F. Van Oystaeyen, Brauer Groups and the Cohomology of Graded Rings, Monographs and Textbooks in Pure and Applied Mathematics, Vol. 121, Marcel Dekker, New York (1988).

[3] F. Castaño Iglesias, J. Gómez Torrecillas and C. Năstăsescu, Separable functors in graded rings, J. Pure Appl. Algebra 127, 219-230 (1998).

[4] L. O. Clark, R. Exel and E. Pardo, A generalised uniqueness theorem and the graded ideal structure of Steinberg algebras, Forum Mathematicum, to appear.

[5] E. C. Dade, Group-Graded Rings and Modules, Math. Z. 174(3), 241-262 (1980).

[6] S. Dǎscălescu, B. Ion, C. Nǎstǎsescu and J. Ríos Montes, Group Gradings on Full Matrix Rings, J. Algebra 220(2), 709-728 (1999).

[7] F. DeMeyer and E. Ingraham, Separable Algebras Over Commutative Rings, Lecture Notes in Mathematics, Vol. 181, Springer-Verlag, Berlin-New York (1971).

[8] M. Dokuchaev, R. Exel and J. J. Simón, Crossed Products by Twisted Partial Actions and Graded Algebras, J. Algebra 320(8), 3278-3310 (2008).

[9] J. Haefner and G. Janusz, Hereditary crossed products, Trans. Amer. Math. Soc. 352(7), 3381-3410 (2000)

[10] J. Haefner and C. J. Pappacena, Strongly graded hereditary orders, Comm. Algebra 29(12), 5535-5542 (2001).

[11] N. Jacobson, Basic Algebra II, W. H. Freeman and Company, New York (1989).

[12] L. Kadison, New Examples of Frobenius Extensions, University Lecture Series, Vol. 14, Amer. Math. Soc., Providence, RI (1999).

[13] T. Y. Lam, A First Course in Noncommutative Rings, Graduate Texts in Mathematics, Vol. 131, SpringerVerlag, New York (1991).

[14] L. Le Bruyn, M. Van den Bergh and F. Van Oystaeyen, Graded Orders, Birkhäuser Boston, Inc., Boston, MA (1988).

[15] P. Lundström, Crossed Product Algebras Defined by Separable Extensions, J. Algebra 283(2), 723-737 (2005).

[16] P. Lundström, Separable Groupoid Rings, Comm. Algebra 34(8), 3029-3041 (2006).

[17] J. Öinert, Simple group graded rings and maximal commutativity. Operator structures and dynamical systems, 159-175, Contemp. Math. 503, Amer. Math. Soc., Providence, RI, 2009.

[18] J. Öinert and P. Lundström, The ideal intersection property for groupoid graded rings, Comm. Algebra 40(5), 1860-1871 (2012). 
[19] Y. Miyashita, On Galois Extensions and Crossed Products, J. Fac. Sci. Hokkaido Univ. Ser. I 21, 97-121 (1970).

[20] C. Năstăsescu and F. Van Oystaeyen, Graded Ring Theory, North-Holland Mathematical Library, Vol. 28, North-Holland Publishing Co., Amsterdam-New York (1982).

[21] C. Năstăsescu, M. Van den Bergh and F. Van Oystaeyen, Separable Functors Applied to Graded Rings, J. Algebra 123(2), 397-413 (1989).

[22] C. Năstăsescu and F. Van Oystaeyen, Methods of graded rings, Lecture Notes in Mathematics, Vol. 1836. Springer-Verlag, Berlin (2004).

[23] M. D. Rafael, Separable functors revisited, Comm. Algebra 18(5), 1445-1459 (1990).

[24] T. Theohari-Apostolidi and H. Vavatsoulas, On the separability of the restriction functor, Algebra Discrete Math. 3, 95-101 (2003). 